\title{
Isolation and Characterization of Plant-Pathogenic Streptomyces Species Associated with Common Scab-Infected Potato Tubers in Newfoundland
}

\author{
Joanna K. Fyans, Luke Bown, and Dawn R. D. Bignell
}

Department of Biology, Memorial University of Newfoundland, St. John's, NL, A1B 3X9, Canada.

Current address of first author: School of Chemistry, The University of Manchester, Oxford Road, Manchester, M13 9PL, UK. Accepted for publication 29 October 2015.

\begin{abstract}
Fyans, J. K., Bown, L., and Bignell, D. R. D. 2016. Isolation and characterization of plant-pathogenic Streptomyces species associated with common scab-infected potato tubers in Newfoundland. Phytopathology 106:123-131.

Potato common scab (CS) is an economically important crop disease that is caused by several members of the genus Streptomyces. In this study, we characterized the plant-pathogenic Streptomyces spp. associated with CS-infected potato tubers harvested in Newfoundland, Canada. A total of 17 pathogenic Streptomyces isolates were recovered from potato scab lesions, of which eight were determined to be most similar to the known CS pathogen S. europaeiscabiei. All eight S. europaeiscabiei

isolates were found to produce the thaxtomin A phytotoxin and to harbor the nec 1 virulence gene, and most also carry the putative virulence gene tomA. The remaining isolates appear to be novel pathogenic species that do not produce thaxtomin $\mathrm{A}$, and only two of these isolates were determined to harbor the necl or tomA genes. Of the non-thaxtominproducing isolates, strain 11-1-2 was shown to exhibit a severe pathogenic phenotype against different plant hosts and to produce a novel, secreted phytotoxic substance. This is the first report documenting the plantpathogenic Streptomyces spp. associated with CS disease in Newfoundland. Furthermore, our findings provide further evidence that phytotoxins other than thaxtomin A may also contribute to the development of CS by Streptomyces spp.
\end{abstract}

Common scab (CS) is an economically important disease of potato (Solanum tuberosum) and is characterized by the presence of necrotic lesions with a corky texture on the potato tuber surface. The lesions can be superficial, erumpent (raised) or deep pitted, and while they can be small and round, they can also coalesce to cover significant areas of the tuber surface (Loria et al. 2006). The incidence and severity of CS disease varies from field to field and within the same field, and from year to year in the same location. There are currently no commercial potato cultivars that are completely resistant to CS disease, and once established in fields the disease is extremely difficult to eradicate or control (Dees and Wanner 2012). CS causes economic losses to growers as the lesions affect the quality and market value of the potato crop. In Canada, it has been estimated that the economic losses from CS during the 2002 growing season were between 15.3 to 17.3 million Canadian dollars (Hill and Lazarovits 2005). There is also evidence that CS can reduce the overall yield of a potato crop and increase the number of smaller tubers in the yield (Hiltunen et al. 2005).

CS is caused by soilborne, filamentous, saprophytic Streptomyces bacteria that have acquired the additional ability to cause plant disease. The best characterized CS-causing pathogen is Streptomyces scabies (syn. scabiei), which is found worldwide and is the predominant CS-causing pathogen in North America (Wanner 2009). A closely related pathogen, S. europaeiscabiei, is frequently found in Europe and has also been isolated in the United States and Canada (Bouchek-Mechiche et al. 2000; Dees et al. 2012, 2013; Flores-González et al. 2008; Wanner 2009). S. turgidiscabies was first isolated from the Japanese island of Hokkaido and is associated with the formation of distinctly erumpent scab lesions

Corresponding author: D. R. D. Bignell; E-mail address: dbignell@mun.ca

First and second authors contributed equally to this study.

http://dx.doi.org/10.1094/PHYTO-05-15-0125-R

(C) 2016 The American Phytopathological Society on potato tubers (Miyajima et al. 1998). It has since been found in Finland, China, Korea, United States, Norway, Sweden, and the UK (Dees et al. 2012, 2013; Kim et al. 1999; Kreuze et al. 1999; Lehtonen et al. 2004; Thwaites et al. 2010; Wanner 2009; Zhao et al. 2008). S. acidiscabies, which causes acid scab (AS) disease, was first isolated from the northeastern United States and has now been found in China, Japan, Korea, Canada, and the UK (Faucher et al. 1992; Lambert and Loria 1989a; Song et al. 2004; St-Onge et al. 2008; Thwaites et al. 2010; Tóth et al. 2001; Zhao et al. 2010). AS is essentially the same disease as CS except that it can occur in acidic pH soils where CS is suppressed (Loria et al. 1997). Other CScausing pathogens that have been described include $S$. stelliscabiei (Bouchek-Mechiche et al. 2000), S. luridiscabiei, S. puniscabiei, S. niveiscabiei (Park et al. 2003), Streptomyces sp. IdahoX (Wanner 2007), Streptomyces sp. DS3024 (Hao et al. 2009), and Streptomyces sp. GK18 (Cao et al. 2012). In addition, some pathogenic strains of S. bottropensis were recently reported (Wanner 2009).

The ability of Streptomyces spp. to cause CS or AS disease is mainly associated with the production of a family of phytotoxic secondary metabolites called the thaxtomins, which are cyclic dipeptides (2,5-diketopiperazines) derived from L-phenylalanine and L-4-nitrotryptophan (Bignell et al. 2014; King and Calhoun 2009). Thaxtomin A, the predominant family member produced by $S$. scabies, $S$. turgidiscabies, and $S$. acidiscabies, functions as an inhibitor of cellulose biosynthesis and may allow penetration of expanding plant tissues during host colonization (Loria et al. 2008). Mutants of $S$. scabies and $S$. acidiscabies that are reduced or defective in thaxtomin A biosynthesis show a corresponding reduction or loss of pathogenicity (Goyer et al. 1998; Healy et al. 2000; Joshi et al. 2007b), indicating that thaxtomin A is an essential virulence factor in these organisms. In addition, S. scabies, $S$. acidiscabies, and $S$. turgidiscabies are known to produce a secreted necrogenic protein called Nec1, which has no homologs in the database or any characterized motifs (Loria et al. 2006). Though not essential for scab disease development, the production of Nec1 appears to contribute to host tissue colonization and may play a role 
in overcoming the host defense response (Joshi et al. 2007a). The tomA gene, which encodes a secreted tomatinase enzyme, is also conserved in S. scabies, S. acidiscabies, and S. turgidiscabies (Kers et al. 2005; Seipke and Loria 2008). Tomatinases belong to a group of enzymes called saponinases, which hydrolyze and detoxify plant-derived antimicrobial compounds called phytoanticipins (VanEtten et al. 1994). Genes encoding saponinases are found in fungal plant pathogens as well as in the tomato bacterial wilt and canker pathogen Clavibacter michiganensis subsp. michiganensis (Loria et al. 2006), and in addition to their role in detoxifying antimicrobial compounds, there is evidence that saponinases can play an indirect role in suppressing the induced plant defense responses (Bouarab et al. 2002; Ito et al. 2004). Although the conservation of tomA in several plant-pathogenic Streptomyces spp. suggests a role in host-pathogen interactions, the precise role remains unclear given that a $\Delta t o m A$ deletion mutant of $S$. scabies was indistinguishable from the wild-type strain in plant bioassays (Seipke and Loria 2008).

Several previous studies have described the pathogenic Streptomyces spp. associated with CS disease in eastern Canada (Faucher et al. 1992; St-Onge et al. 2008; Wanner 2009); however, to our knowledge, there is no information regarding the Streptomyces spp. that are responsible for the disease on the island of Newfoundland. The objective of this study was therefore to isolate and characterize the plant-pathogenic Streptomyces spp. that are associated with potato CS disease in Newfoundland.

\section{MATERIALS AND METHODS}

Streptomyces spp. used in this study. The previously described pathogenic Streptomyces spp. S. scabies 87-22, S. acidiscabies 84.104, and S. turgidiscabies Car8 (Lambert and Loria 1989a,b; Loria et al. 1995; Miyajima et al. 1998) as well as the nonpathogenic strain $S$. scabies $\Delta t x t A / \Delta c f a 6$ (Bignell et al. 2010) were used as reference strains in this study.

Isolation of Streptomyces spp. from potato scab lesions. Streptomycetes were isolated from scabby potato tubers that were harvested in Newfoundland in 2011 and 2012. The potatoes were harvested on the Avalon Peninsula from a single location in 2011 and from three different locations in 2012. The isolation procedure was as described previously (Wanner 2004) with some modifications. Briefly, a small piece of tuber containing a scab lesion was excised and surface-sterilized using 1.5\%, vol/vol, Chlorox bleach for $2 \mathrm{~min}$, after which the tissue was rinsed 10 times with sterile high-performance liquid chromatography (HPLC)-grade water. Then, the tissue was homogenized in $0.5 \mathrm{ml}$ of sterile water, and the homogenate was incubated at $55^{\circ} \mathrm{C}$ for $30 \mathrm{~min}$ to eliminate any competing rhizobacteria. Next, the homogenate was diluted 100 fold and was plated onto agar $(1.5 \%, \mathrm{wt} / \mathrm{vol})$ water plates containing nalidixic acid at $25 \mu \mathrm{g} / \mathrm{ml}$ and nystatin at $50 \mu \mathrm{g} / \mathrm{ml}$. The plates were incubated at $28^{\circ} \mathrm{C}$ for up to 1 month, after which the Streptomyceslike colonies were picked and subjected to several rounds of culturing on the International Streptomyces project medium 4 (ISP-4; BD Biosciences, Mississauga, ON) agar plates containing nalidixic acid and nystatin in order to obtain pure cultures of each isolate. Finally, spore or mycelial glycerol stocks of each Streptomyces-like isolate were prepared from a single, well-separated colony as described previously (Kieser et al. 2000) and were stored at $-80^{\circ} \mathrm{C}$.

Morphological and physiological characterization. Streptomyces strains were cultured on ISP-4 for 14 to 15 days at $28^{\circ} \mathrm{C}$, after which the aerial hyphae color of each strain was recorded. Production of the brown secreted pigment melanin was assessed by culturing the isolates in trypticase soy broth (TSB; BD Biosciences) for 2 to 4 days at $28^{\circ} \mathrm{C}$. To test for utilization of different carbon sources, spores of Streptomyces strains were scraped from 10day-old potato mash agar plates (Fyans et al. 2015), washed several times with sterile water, and then resuspended in water. The suspensions $(25 \mu \mathrm{l})$ were spotted onto plates of the International
Streptomyces project medium 9 (ISP-9; Himedia Laboratories, Mumbai, India) containing transaconitic acid, D-(+)-trehalose, betaine, and methyl- $\alpha$-D-galactopyranoside (OMPG) (Sigma Aldrich Canada, Oakville, ON) as carbon sources (1\%, wt/vol, final concentration). Growth was assessed on the plates following incubation at $28^{\circ} \mathrm{C}$ for up to 7 days. Medium containing D-glucose as the carbon source served as a positive control while medium containing water in place of a carbon source served as a negative control. Sensitivity to penicillin G was tested by spotting Streptomyces spore suspensions onto nutrient agar (BD Biosciences) containing $10 \mathrm{IU} / \mathrm{ml}$ of penicillin G (Sigma Aldrich Canada). Medium lacking antibiotic served as a negative control.

Pathogenicity testing. The pathogenicity of the Streptomyces isolates was assessed using a radish seedling bioassay as described previously (Bignell et al. 2010) with some modifications. Briefly, Streptomyces isolates were cultured in triplicate in $5 \mathrm{ml}$ of oat bran broth (OBB) (Johnson et al. 2007) medium for 7 days at $25^{\circ} \mathrm{C}$ with shaking (125 rpm) in six-well tissue culture plates, after which the mycelia were harvested, washed twice with sterile water, and resuspended in $5 \mathrm{ml}$ of sterile water. Radish seeds ('Cherry Belle'; Heritage Harvest Seed, Carmen, MB) were surface sterilized by treating with $70 \%$, vol/vol, ethanol for $5 \mathrm{~min}$ followed by $15 \%, \mathrm{vol} / \mathrm{vol}$, Chlorox bleach for $10 \mathrm{~min}$. The seeds were rinsed several times with sterile water and were then germinated by incubating at room temperature $\left(22\right.$ to $24^{\circ} \mathrm{C}$ ) for $24 \mathrm{~h}$ in the dark in a sealed Petri dish containing moistened filter paper. Germinated seeds were placed into wells (13 mm in diameter) in $1.5 \%$, wt/vol, agar-water plates and were inoculated with the Streptomyces mycelial suspensions $(200 \mu \mathrm{l})$. A total of six germinated seeds were inoculated with each mycelial suspension (for a total of 18 seeds per Streptomyces isolate), and control plants were inoculated with known pathogenic Streptomyces species (S. scabies 87-22, S. acidiscabies 84-104, and/or S. turgidiscabies Car8) or were treated with a nonpathogenic strain (S. scabies $\Delta t x t A / \Delta c f a 6$ ) or with water (uninoculated control). The plates were wrapped with parafilm and incubated at $22 \pm 2^{\circ} \mathrm{C}$ under a 16-h photoperiod for 7 days. The total plant size (root + shoot) was measured and averaged for each treatment, and the Student's $t$ test was used to identify statistically significant differences among the treatments compared with the uninoculated control $(P<0.05)$.

The pathogenicity of the 11-1-2 isolate was further characterized using a potato tuber slice bioassay as described previously (Loria et al. 1995). Potato tuber disks were aseptically prepared and were placed onto moistened filter paper (Whatman 3M, $90 \mathrm{~mm}$ ) in deep $(25 \mathrm{~mm})$ Petri dishes. The tuber disks (5 in total) were then inoculated with mycelia $(25 \mu \mathrm{l})$ prepared from OBB cultures. Negative control disks were treated with water while positive control disks were treated with mycelia of the S. turgidiscabies Car8 strain. The Petri dishes were wrapped with Parafilm and were incubated in the dark at $22 \pm 2^{\circ} \mathrm{C}$ for 7 days, after which the potato slices were photographed.

A leaf infiltration bioassay was also performed to assess the pathogenicity of the 11-1-2 strain. Leaves of 6-week-old Nicotiana benthamiana plants were infiltrated with a spore suspension of the 11-1-2 strain in water. Spores of the $S$. scabies 87-22 strain as well as other Streptomyces strains were included in the analysis, and water served as the negative control. A total of two to three plants were used for each infiltration experiment, and two to three leaves per plant were infiltrated with each sample. Following infiltration, the plants were incubated at $22 \pm 2^{\circ} \mathrm{C}$ under a 16-h photoperiod for 11 days, after which the leaves were removed and photographed.

Bioactivity of the secreted 11-1-2 phytotoxin. Strain 11-1-2 was cultured in OBB as described above, after which the culture supernatants were harvested and filter-sterilized using $0.45 \mu \mathrm{m}$ syringe filters. Aliquots $(25 \mu \mathrm{l})$ were then tested for bioactivity using the radish seedling bioassay and the leaf infiltration bioassay as described above, except that the $N$. benthamiana leaves used in the infiltration assay were photographed after 5 days incubation. Uninoculated OBB medium served as the negative control while 
filter-sterilized OBB culture supernatants of S. scabies 87-22 served as the positive control. To determine whether the phytotoxic substance produced by strain 11-1-2 is soluble in organic solvent, the OBB culture supernatants $(1 \mathrm{ml})$ were extracted three times with $0.5 \mathrm{ml}$ of ethyl acetate, and the resulting extracts were combined, dried down, and resuspended in $0.5 \mathrm{ml}$ of $100 \%$ methanol. The extracts $(25 \mu \mathrm{l})$ were then tested in the potato tuber disk assay as described above, with $100 \%$ methanol serving as the negative control and extract from OBB cultures of S. scabies 87-22 serving as the positive control.

Production of thaxtomin A. Thaxtomin A production was assessed by culturing the Streptomyces isolates in OBB medium as described above. The supernatants were harvested following centrifugation of the cultures, and the remaining mycelial pellets were dried for $48 \mathrm{~h}$ at $50^{\circ} \mathrm{C}$ and were weighed. A $1-\mathrm{ml}$ aliquot of each supernatant was extracted with $0.5 \mathrm{ml}$ of ethyl acetate, and the resulting extracts were dried by evaporation, resuspended in $100 \mu \mathrm{l}$ of $100 \%$ HPLC-grade methanol and filtered through a $0.2-\mu \mathrm{m}$ syringe filter. Detection of thaxtomin A in the extracts was by reverse phase (RP)-HPLC using an Agilent 1260 Infinity Quaternary LC system (Agilent Technologies Canada Inc., Mississauga, ON). Samples (5 $\mu$ l) were loaded onto a Poroshell
120 EC-C18 column $(4.6 \times 50 \mathrm{~mm}, 2.7 \mu \mathrm{m}$ particle size; Agilent Technologies Canada Inc.) and were eluted with an isocratic mobile phase consisting of $30 \%$ acetonitrile (ACN) and $70 \%$ water at a constant flow rate of $1 \mathrm{ml} / \mathrm{min}$. Metabolites were monitored using a detection wavelength of $380 \mathrm{~nm}$, and the ChemStation software version B.04.03 (Agilent Technologies Canada Inc.) was used for data acquisition and analysis. Quantification of thaxtomin A production was performed by constructing a standard curve using known amounts of a pure, authentic thaxtomin A standard (Santa Cruz Biotechnology Inc., Dallas, TX), and the results were reported as micrograms of thaxtomin A produced per milligram of dried cells.

Production of concanamycin A and borrelidin. Detection of concanamycin A and borrelidin production was by RP-HPLC analysis of ethyl acetate culture extracts as described previously (Natsume et al. 1998; Olano et al. 2004). Briefly, samples (5 $\mu \mathrm{l}$ ) were loaded onto a Poroshell 120 EC-C18 RP column held at $40^{\circ} \mathrm{C}$. For detection of concanamycin A, an isocratic mobile phase consisting of $30 \% \mathrm{ACN}$ and $70 \%$ water was used at a constant flow rate of $1 \mathrm{ml} / \mathrm{min}$. Metabolites were monitored using a detection wavelength of $245 \mathrm{~nm}$, and a pure, authentic standard of concanamycin A (Sigma Aldrich Canada, Oakville, ON) was used for phytotoxin

TABLE 1. Primers used for polymerase chain reaction and sequence analysis of the Newfoundland pathogenic Streptomyces isolates

\begin{tabular}{|c|c|c|c|}
\hline Gene/region & Primers & Nucleotide sequence $\left(5^{\prime}-3^{\prime}\right)$ & Reference \\
\hline \multirow[t]{2}{*}{$t x t A$} & JKF_txtA_for & ACACTCGCGCTGTTCGACCG & This study \\
\hline & JKF_txtA_rev & ACAGCCACGACAGCGGCATC & \\
\hline \multirow[t]{2}{*}{$t x t D$} & JKF_txtD_for & ACTGGAACAGCCTGCGCGTC & This study \\
\hline & JKF_txtD_rev & CCGTGCCCATGTACCAGCCG & \\
\hline \multirow[t]{2}{*}{ necl } & JKF_nec1_for & ATGAGCGCGAACGGAAGCCCCGGA & Bukhalid et al. 1998 \\
\hline & JKF_nec1_rev & GCAGGTCGTCACGAAGGATCG & \\
\hline \multirow[t]{2}{*}{ tomA } & JKF_tomA_for & GAGGCGTTGGTGGAGTTCTA & St-Onge et al. 2008 \\
\hline & JKF_tomA_rev & TTGGGGTTGTACTCCTCGTC & \\
\hline \multirow[t]{2}{*}{ гров } & rpoBF1 & TTCATGGACCAGAACAACC & Guo et al. 2008 \\
\hline & rpoBR1 & CGTAGTTGTGACCCTCCC & \\
\hline \multirow[t]{2}{*}{$16 \mathrm{~S}-23 \mathrm{~S}$ rDNA ITS } & ITS_2F & AAACTTGGCCACAGATGCTC & Song et al. 2004 \\
\hline & ITS_2R & GTCAAGTCATCATGCCCCTT & \\
\hline BOX elements & BOX̄A1R & CTACGGCAAGGCGACGCTGACG & Clark et al. 1998 \\
\hline \multirow[t]{2}{*}{ ERIC elements } & ERIC2 & AAGTAAGTGACTGGGGTGAGCG & Clark et al. 1998 \\
\hline & ERIC1R & ATGTAAGCTCCTGGGGATTCAC & \\
\hline
\end{tabular}

TABLE 2. Phenotypic and genotypic characterization of the Newfoundland pathogenic Streptomyces isolates

\begin{tabular}{|c|c|c|c|c|c|c|c|c|}
\hline \multirow[b]{2}{*}{ Isolate number } & \multirow[b]{2}{*}{ Aerial hyphae color ${ }^{\mathrm{a}}$} & \multirow[b]{2}{*}{ Melanin poduction ${ }^{\mathrm{b}}$} & \multirow[b]{2}{*}{ Degree of pathogenicity ${ }^{c}$} & \multicolumn{4}{|c|}{$\begin{array}{l}\text { Known/predicted } \\
\text { virulence genes }^{\mathrm{d}}\end{array}$} & \multirow[b]{2}{*}{ Thaxtomin A production } \\
\hline & & & & $t x t A$ & $t x t D$ & necl & tomA & \\
\hline $11-1-2$ & Gray & - & $++^{*}$ & - & - & - & - & - \\
\hline $11-1-7$ & Gray & - & ++ & - & - & - & + & - \\
\hline $11-1-10$ & Green & + & + & - & - & + & - & - \\
\hline $11-2-1$ & Gray & - & + & - & - & - & - & - \\
\hline $11-2-4$ & Gray & - & ++ & - & - & - & - & - \\
\hline $12-2-3$ & Brown & - & + & - & - & - & - & - \\
\hline $12-4-1$ & Gray & + & $++^{*}$ & + & + & + & - & + \\
\hline $12-11-1$ & Gray & + & $++^{*}$ & + & + & + & + & + \\
\hline $12-11-3$ & Gray & + & $++^{*}$ & + & + & + & + & + \\
\hline $12-11-5$ & Gray & + & ++ & - & - & - & - & - \\
\hline $12-15-1$ & Gray & - & + & - & - & - & - & - \\
\hline LF12-2-1 & Gray & + & $++^{*}$ & + & + & + & + & + \\
\hline LF12-2-2 & Gray & + & $++^{*}$ & + & + & + & + & + \\
\hline LF12-2-3A & Gray & + & $++^{*}$ & + & + & + & + & + \\
\hline LF12-2-4 & Gray & + & $++^{*}$ & + & + & + & + & + \\
\hline LF12-3-2 & Gray & + & $++^{*}$ & + & + & + & + & + \\
\hline LF12-4-3 & Gray & - & ++ & - & - & - & - & - \\
\hline
\end{tabular}

${ }^{a}$ Aerial hyphae color was assessed on the International Streptomyces Project medium 4 (ISP-4) after 14 to 15 days incubation at $28^{\circ} \mathrm{C}$.

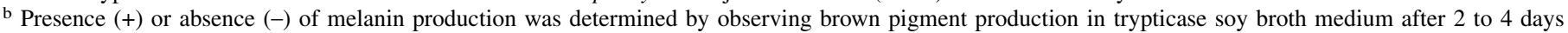
incubation at $28^{\circ} \mathrm{C}$.

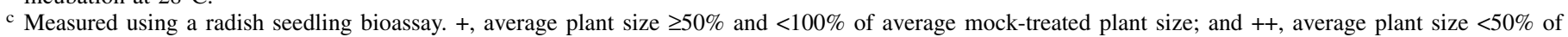
average mock-treated plant size. * Indicates that the degree of pathogenicity observed was comparable to that of the control strains $S$. scabies $87-22$, S. acidiscabies 84.104, and S. turgidiscabies Car8.

d Presence (+) or absence (-) of known or predicted virulence genes was determined using polymerase chain reaction.

e Production $(+)$ or lack thereof $(-)$ of thaxtomin A was determined by reverse-phase high performance liquid chromatography analysis of culture extracts. 
identification in the culture extracts. For detection of borrelidin, the solvent system consisted of $100 \%$ water as mobile phase A and $100 \%$ ACN as mobile phase B, both containing $0.1 \%$ formic acid. A linear gradient from $10 \% \mathrm{~B}$ to $100 \% \mathrm{~B}$ over $5.8 \mathrm{~min}$ was applied at a constant flow rate of $1 \mathrm{ml} / \mathrm{min}$. Metabolites were monitored at $257 \mathrm{~nm}$, and a pure, authentic standard of borrelidin (kindly provided by Kenji Arakawa, Hiroshima University) was used for identification of the phytotoxin in the culture extracts.

Analysis of borrelidin production was also conducted using liquid chromatography-mass spectrometry (LC-MS) with an Agilent 1100 series HPLC system (Agilent Technologies Canada Inc.) interfaced to a Waters G1946A single quadrupole mass spectrometer (Waters Canada, Mississauga, ON). Samples $(15 \mu \mathrm{l})$ were loaded onto a Zorbax SB-C18 column $(4.6 \times 150 \mathrm{~mm}, 5 \mu \mathrm{m}$ particle size; Agilent Technologies Canada Inc.) and were eluted using a linear gradient from 10 to $100 \%$ ACN over $17.4 \mathrm{~min}$ at a constant flow rate of $1 \mathrm{ml} / \mathrm{min}$. Detection was by UV radiation $(257 \mathrm{~nm})$ and electrospray ionization MS in negative ion mode.

Genotypic characterization. Genomic DNA was prepared from the Streptomyces isolates using the DNeasy Blood and Tissue Kit as per the manufacturer's protocol (Qiagen Inc. Canada, Toronto, ON). Primers used for PCR or sequencing were purchased from Integrated DNA Technologies (IDT; Coralville, IA) and are listed in Table 1. Amplification of the rpoB, txtA, txtD, necl, and tomA genes and the 16S-23S rDNA internally transcribed spacer (ITS) region was carried out using Phusion DNA polymerase (New England Biolabs Canada, Whitby, ON) or Taq DNA polymerase (Thermo Fisher Scientific Canada, Ottawa, ON) according to the manufacturer's instructions, except that DMSO (5\%, vol/vol, final concentration) was included in the reactions. Sequencing of the $r p o B$ PCR products was performed at The Centre for Applied Genomics (TCAG) (Toronto, ON). RFLP analysis of the amplified 16S-23S rDNA ITS region was performed using the Hpy99I restriction enzyme (New England Biolabs Canada) to distinguish $S$. scabies from $S$. europaeiscabiei as described previously (Flores-González et al. 2008). Repetitive element PCR (Rep-PCR) fingerprinting was performed using the BOXA1R primer or the ERIC2/ERIC1R primers (Table 1). Reactions $(25 \mu \mathrm{l})$ contained 1× Taq Buffer with $\mathrm{KCl}$ (Thermo Fisher Scientific), $4 \mathrm{mM}$ (for BOX-PCR) or $6 \mathrm{mM}$ (for ERIC-PCR) $\mathrm{MgCl}_{2}, 0.75 \mathrm{mM}$

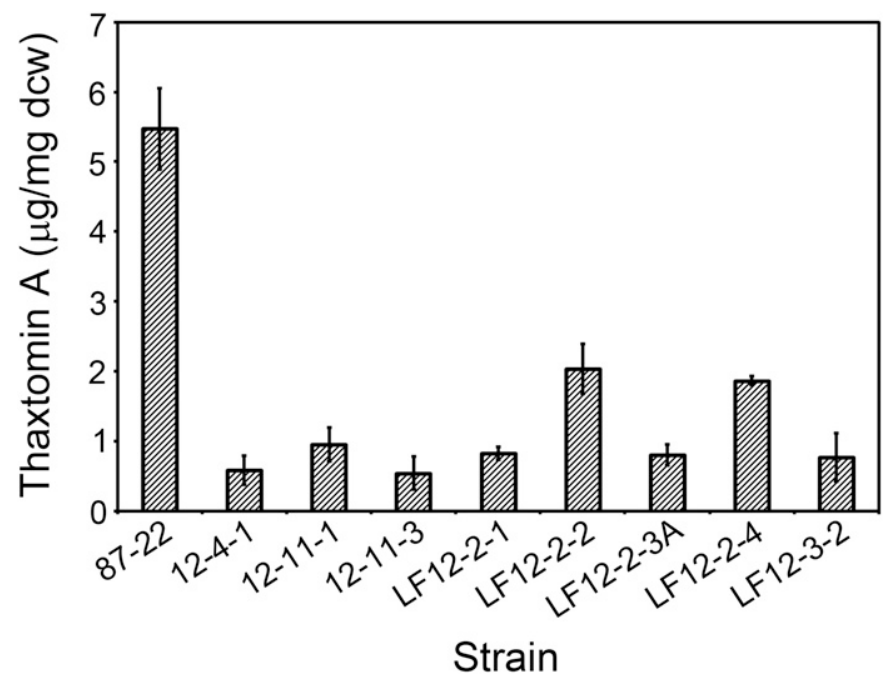

Fig. 1. Production of thaxtomin A by plant-pathogenic Streptomyces spp. isolated in this study. Supernatants from triplicate oat bran broth cultures of S. scabies $87-22$ and of the pathogenic isolates were extracted with ethyl acetate, and the resulting extracts were analyzed by reversed phase-high performance liquid chromatography. The average production level (micrograms of thaxtomin A per milligrams of dry cell weight) for each strain is shown, and the errors bars represent the standard deviation from the mean.
dNTPs (New England Biolabs Canada), 10\%, vol/vol, DMSO, $1 \mu \mathrm{M}$ of each primer ( $2 \mu \mathrm{M}$ of the BOXA1R primer), $1.25 \mathrm{U}$ of Taq DNA polymerase (Thermo Fisher Scientific Canada), and $50 \mathrm{ng}$ of genomic DNA. The cycling conditions were as follows: an initial denaturation step at $95^{\circ} \mathrm{C}$ for 5 min followed by 35 cycles of denaturation at $95^{\circ} \mathrm{C}$ for $1 \mathrm{~min}$, primer annealing at $50^{\circ} \mathrm{C}$ for $1 \mathrm{~min}$, and extension at $72^{\circ} \mathrm{C}$ for $4 \mathrm{~min}$. The resulting products were analyzed by gel electrophoresis using a $1.5 \%$, wt/vol, agarose gel and $1 \times$ TBE buffer.

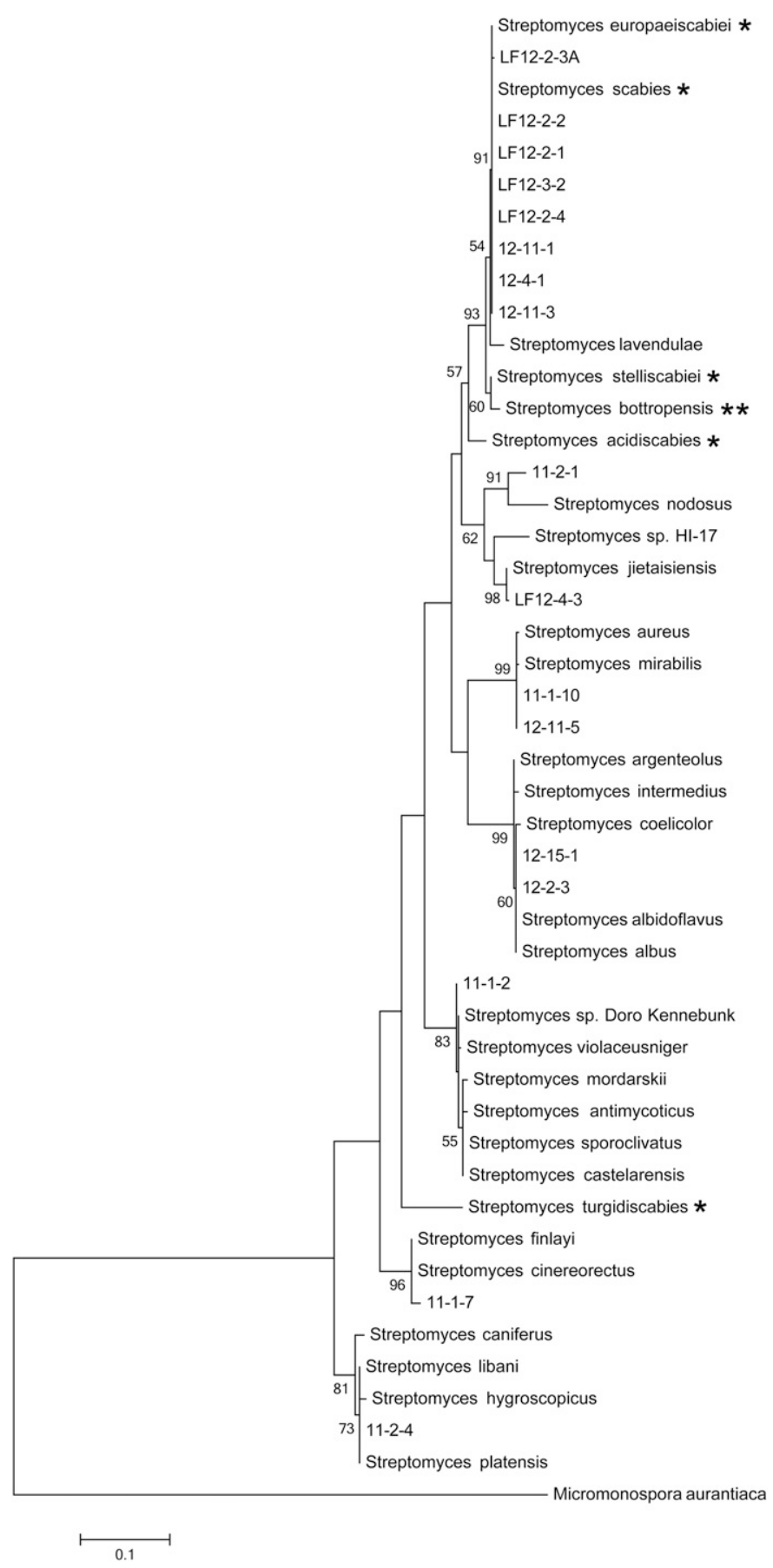

Fig. 2. Phylogenetic analysis of plant-pathogenic Streptomyces spp. isolated in this study. The tree is based on the partial rроB gene sequence from the pathogenic isolates and from Streptomyces spp. in the database. Bootstrap values $\geq 50 \%$ for 1,000 repetitions are shown. The scale bar indicates the number of nucleotide substitutions per site. Known pathogenic Streptomyces spp. are indicated with *, and nonpathogenic species that have been reported to have some pathogenic strains are indicated with **. The rpoB sequence from Micromonospora aurantiaca was included as an outgroup. 
Phylogenetic analyses. Alignment of the $r p o B$ sequences was performed using the Geneious Pro software version 6.1.2 (Biomatters Ltd., San Francisco, CA). The phylogenetic tree was generated using the MEGA 6.0.6 program (Tamura et al. 2011) with the maximum likelihood method. The significance of the branch order was assessed using the bootstrapping method with 1,000 repetitions.

Accession numbers. The partial $r p o B$ sequences generated in this study are deposited in GenBank under the accession numbers KR817679 to KR817695.

\section{RESULTS AND DISCUSSION}

Potato tubers harvested in Newfoundland in 2011 and 2012 and displaying typical CS disease symptoms were used to isolate pathogenic Streptomyces spp. as described in the Materials and Methods. A total of 52 bacterial strains resembling Streptomyces spp. in appearance were recovered from scab lesions, of which 17 (33\%) were determined to have a plant-pathogenic phenotype when tested in a radish seedling bioassay (Table 2). The degree of pathogenicity varied among the different isolates, with several isolates causing severe stunting of radish seedlings in a similar manner as the known pathogenic strains S. scabies 87-22, S. acidiscabies 84.104, and S. turgidiscabies Car8 (Table 2). Like $S$. scabies, most of the pathogenic isolates produced gray spores
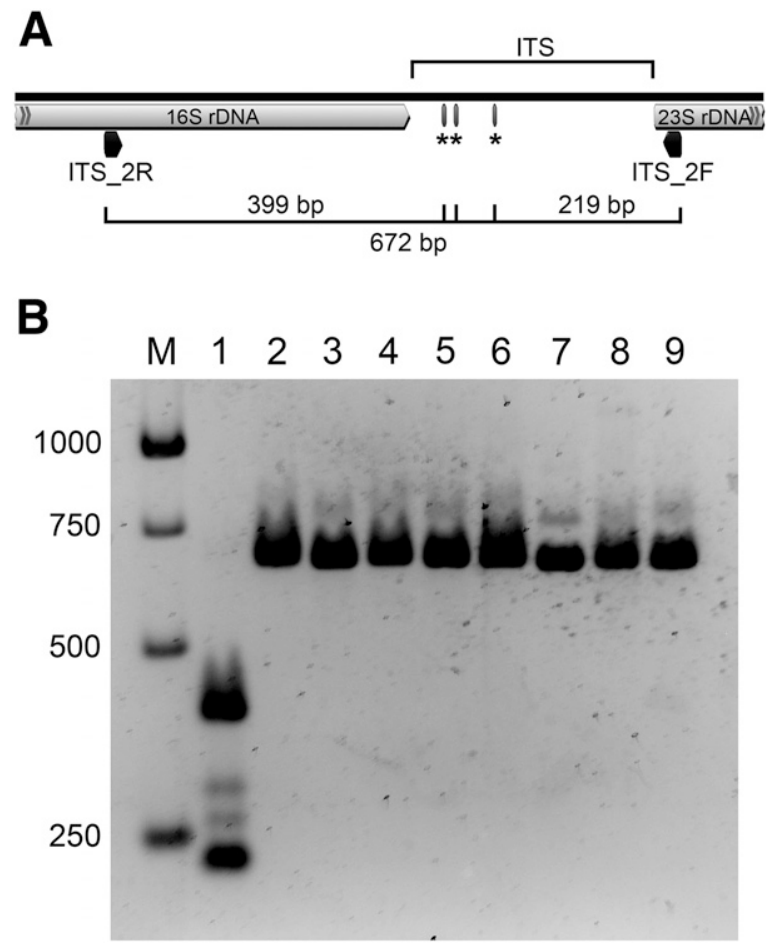

Fig. 3. Restriction fragment length polymorphic analysis of the $16 \mathrm{~S}-23 \mathrm{~S}$ rDNA internally transcribed spacer (ITS) sequence in the thaxtomin A-producing Streptomyces spp. isolated in this study. A, Diagram of the genomic 16S-23S rDNA ITS region in S. scabies 87-22. The primers used for polymerase chain reaction (PCR) amplification of the ITS region are shown as well as the Hpy99I restriction sites (indicated by *) that are present within the amplified region. The size of the amplified PCR product as well as the main fragment sizes expected following digestion with Hpy99I are also indicated. B, Agarose gel electrophoresis of the PCR-amplified 16S-23S rDNA ITS region following digestion with Hpy99I. Electrophoresis was conducted using a 2\%, wt/vol, agarose gel and $1 \times$ TBE buffer, and DNA bands were visualized under UV light following staining with ethidium bromide. Lane assignments are as follows: M, 1-kb DNA ladder (Frogga Bio Inc.); 1, S. scabies 87-22; 2, strain LF12-2-3; 3, strain LF12-2-1; 4, strain LF12-2-3A; 5, strain LF12-2-4; 6, strain LF12-3-2; 7, strain 12-11-1; 8, strain 12-11-3; and 9, strain 12-4-1. The sizes (in bp) of the DNA ladder bands are indicated. when cultured on ISP-4 agar medium, and 10 of the isolates were determined to produce the secreted brown pigment melanin in TSB (Table 2). Production of melanin is a characteristic that has been reported for both $S$. scabies and $S$. europaeiscabiei (Bouchek-Mechiche et al. 2000; Lambert and Loria 1989b). Interestingly, only eight $(47 \%)$ of the pathogenic isolates were determined to produce the known virulence determinant thaxtomin A under the culture conditions used (Table 2 and Fig. 1). All of the thaxtomin-producing strains were confirmed to harbor the txtA and $t x t D$ thaxtomin biosynthetic genes as well as the necl virulence gene, and seven of these strains were also determined to contain the putative tomA virulence gene (Table 2). In contrast, only two of the non-thaxtomin-producing strains were found to contain either the necl or the tomA gene (Table 2). This suggests that novel virulence factors may be contributing to the plantpathogenic phenotype of these strains.

To determine the closest relative of each of the pathogenic isolates, a portion of the $r p o B$ gene from each strain was PCR-amplified and sequenced, and the resulting sequences were
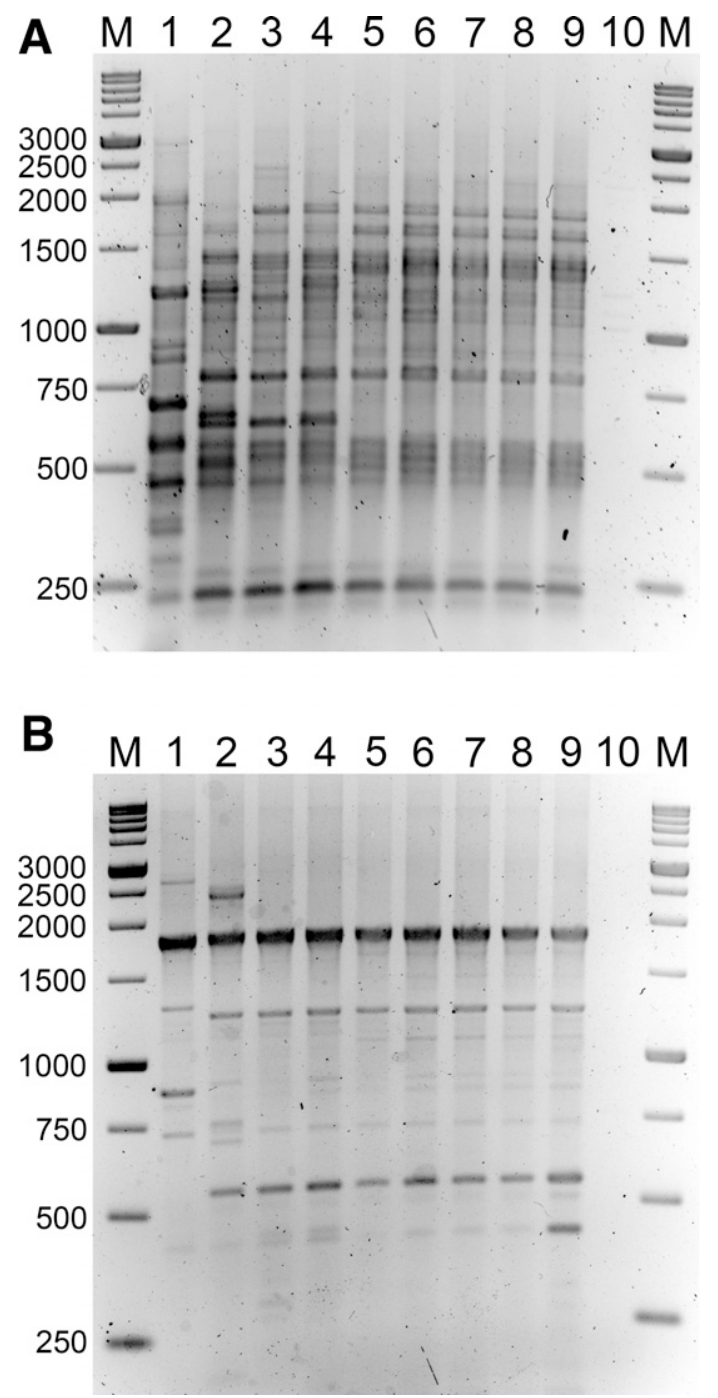

Fig. 4. Rep-polymerase chain reaction (PCR) analysis of the thaxtomin A-producing Streptomyces spp. isolated in this study. A, BOX-PCR and B, ERICPCR fingerprints following electrophoresis on a $1.5 \%$, wt/vol, agarose gel in $1 \times$ TBE buffer. DNA bands were visualized under UV light following staining with ethidium bromide. Lane assignments are as follows: M, $1 \mathrm{~kb}$ DNA ladder (Frogga Bio Inc.); 1, S. scabies 87-22; 2, strain 12-4-1; 3, strain 12-11-1; 4, strain 12-11-3; 5, strain LF12-2-1; 6, strain LF12-2-2; 7, strain LF2-2-3A; 8, strain LF12-2-4; 9, strain LF12-3-2; and 10, water control. The sizes (in bp) of the DNA ladder bands are indicated. 
used to construct a phylogenetic tree. As shown in Figure 2, the nonthaxtomin-producing pathogenic isolates were found scattered throughout the tree and did not cluster with any of the known scab-causing pathogens, suggesting that these isolates may represent novel pathogenic species. On the other hand, the thaxtomin A-producing isolates all clustered together with $S$. scabies and S. europaeiscabiei, indicating that the isolates likely belong to one of these species, a result that is consistent with the observed phenotypic characteristics (Table 2). To further investigate the identity of the thaxtomin-producing isolates, the strains were cultured on ISP-9 medium containing betaine, transaconitic acid, D-(+)-trehalose and OMPG as carbon sources. It has been reported (Bouchek-Mechiche et al. 2000; Flores-González et al. 2008) that in contrast to $S$. scabies, S. europaeiscabiei can utilize transaconitate, D-(+)-trehalose and OMPG but not betaine as carbon sources. However, we found that all of the thaxtominproducing isolates as well as the $S$. scabies 87-22 reference strain could grow on all of the ISP-9 test plates as well as on the negative control plate containing no added carbon source (data not shown). In addition, the test and reference strains all grew on nutrient agar containing $10 \mathrm{IU} / \mathrm{ml}$ of penicillin $\mathrm{G}$ (data not shown) even though it has been reported that $S$. scabies, but not $S$. europaeiscabiei, is sensitive to penicillin $\mathrm{G}$ at that concentration (Bouchek-Mechiche et al. 2000; Lambert and Loria 1989b). We then performed RFLP analysis of the PCR-amplified 16S-23S rDNA ITS region using the Hpy99I restriction enzyme, which cuts within the S. scabies $16 \mathrm{~S}-23 \mathrm{~S}$ rDNA ITS region and not at all within the corresponding region in S. europaeiscabiei (Flores-González et al. 2008). As shown in Figure 3, the $S$. scabies 87-22 ITS PCR product was, as expected, restricted with Hpy99I, whereas none of the ITS PCR products from the thaxtomin-producing isolates were restricted with this enzyme, suggesting that the isolates most likely belong to
S. europaeiscabiei. Further analysis using BOX- and ERIC-PCR fingerprinting confirmed that the isolates are all genetically distinct from the $S$. scabies 87-22 reference strain (Fig. 4). It is noteworthy that although the BOX and ERIC band patterns were very similar among the thaxtomin-producing isolates, some differences were observed (Fig. 4) suggesting that there is some genetic heterogeneity among the isolates.

Given that previous reports have shown that S. scabies is commonly associated with CS disease in eastern Canada (Faucher et al. 1992; St-Onge et al. 2008; Wanner 2009), the absence of S. scabies from the Newfoundland pathogenic isolates was surprising. This may be due to the limited number of sites that were used for sampling in this study, and a more extensive survey of pathogenic isolates from different regions of the island may be needed in order to detect the presence of $S$. scabies. S. europaeiscabiei is known to be the predominant pathogen in Europe (Dees and Wanner 2012), and a large-scale survey by Wanner (2009) demonstrated that it is also common in North America and may be the predominant pathogenic species in the Canadian provinces of Ontario and Prince Edward Island (Wanner 2009). The results of our study suggest that S. europaeiscabiei may also be common in Newfoundland, though further studies will be needed to confirm this.

The 11-1-2 strain was of particular interest to us as it was the only non-thaxtomin A-producing strain that exhibited a severe pathogenic phenotype in the radish seedling bioassay (Table 2). Further characterization of the pathogenic phenotype of this strain was performed using a potato tuber disk assay, which demonstrated that the strain could cause significant pitting of potato tuber tissue (Fig. 5A). In addition, an $N$. benthamiana leaf infiltration assay showed that strain 11-1-2 could induce severe necrosis of the leaf tissue in the region of infiltration (Fig. 5D). Interestingly, this did not occur when $S$. turgidiscabies Car8 was infiltrated into the leaf
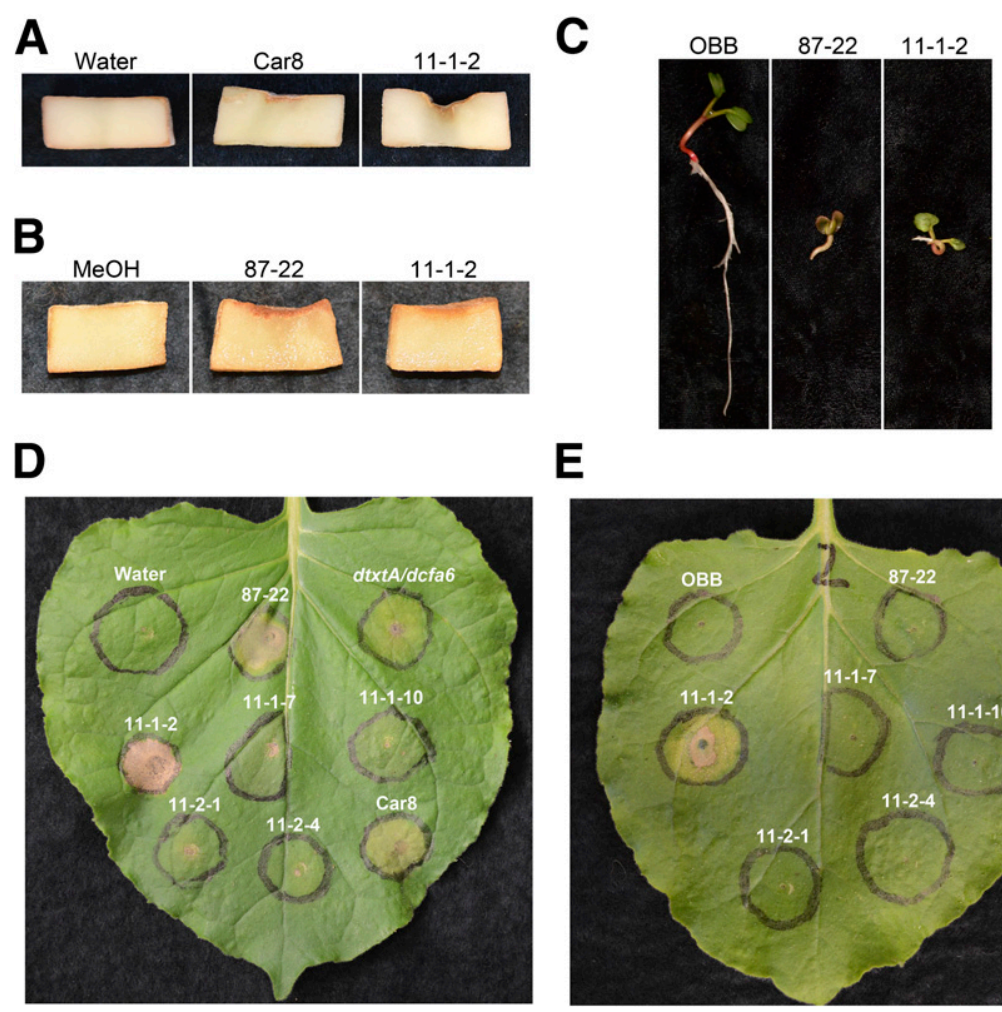

\section{$\mathbf{E}$}

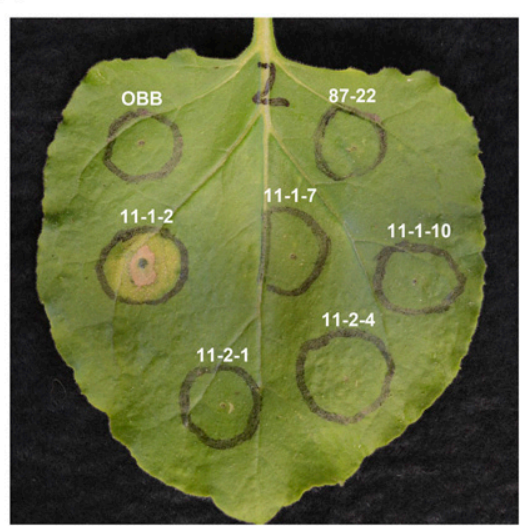

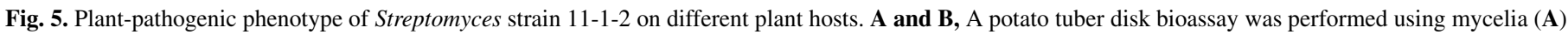

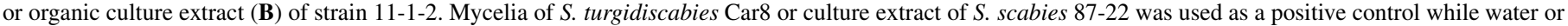

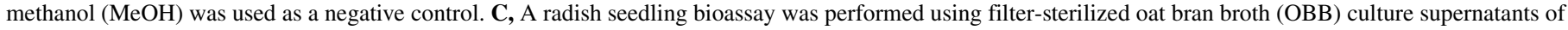

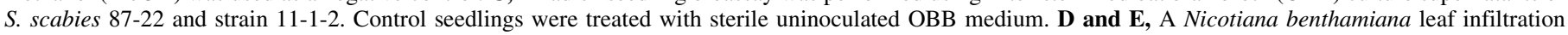

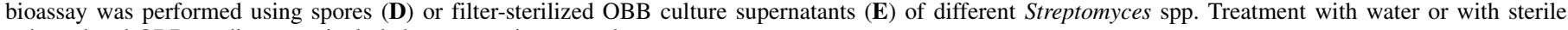
uninoculated OBB medium was included as a negative control. 
tissue, and although tissue necrosis was observed when $S$. scabies 87-22 was infiltrated, the degree of necrosis was consistently lower than that observed with strain 11-1-2 (Fig. 5D). To determine whether a secreted phytotoxin contributes to the plant-pathogenic phenotype of strain 11-1-2, supernatants of OBB-grown 11-1-2 were harvested, filter-sterilized, and tested in different plant bioassays. Figure 5C shows that in the radish seedling bioassay, the 11-1-2 culture supernatant caused seedling stunting in a similar manner as the S. scabies 87-22 culture supernatant, and it was consistently the only supernatant that produced chlorosis and necrosis in the $N$. benthamiana leaf infiltration assay (Fig. 5E). This latter observation was of particular interest as it suggests that another virulence factor besides thaxtomin $\mathrm{A}$ is responsible for the observed tissue necrosis caused by the S. scabies 87-22 strain (Fig. 5D). Furthermore, when the 11-1-2 culture supernatant was subjected to extraction with ethyl acetate, the resulting extract could cause pitting and necrosis of the potato tuber tissue (Fig. 5B), indicating that the secreted phytotoxic substance produced by strain 11-1-2 is soluble in organic solvent.

In addition to thaxtomin A, S. scabies is known to produce concanamycin A, which displays antifungal and phytotoxic activities (Kinashi et al. 1984; Natsume et al. 1996, 1998). We hypothesized that perhaps strain 11-1-2 can also produce this phytotoxin; however, analysis of the 11-1-2 organic culture extracts using RP-HPLC could not detect the presence of concanamycin A in the extracts (Fig. 6A). It was also recently reported that a Streptomyces strain causing deep-pitted lesions on potato tubers does not produce thaxtomins but instead produces an 18-membered macrolide called borrelidin, which in purified form could induce necrosis of potato tuber slices and inhibit the growth of roots and shoots of radish
A
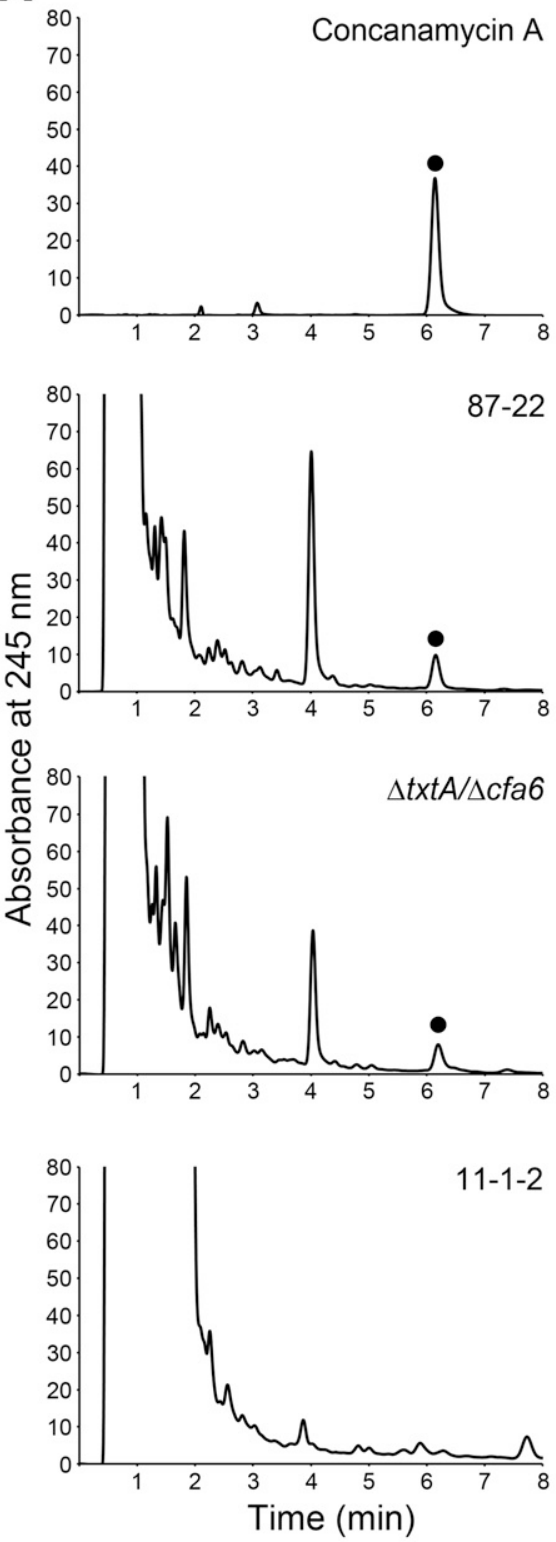
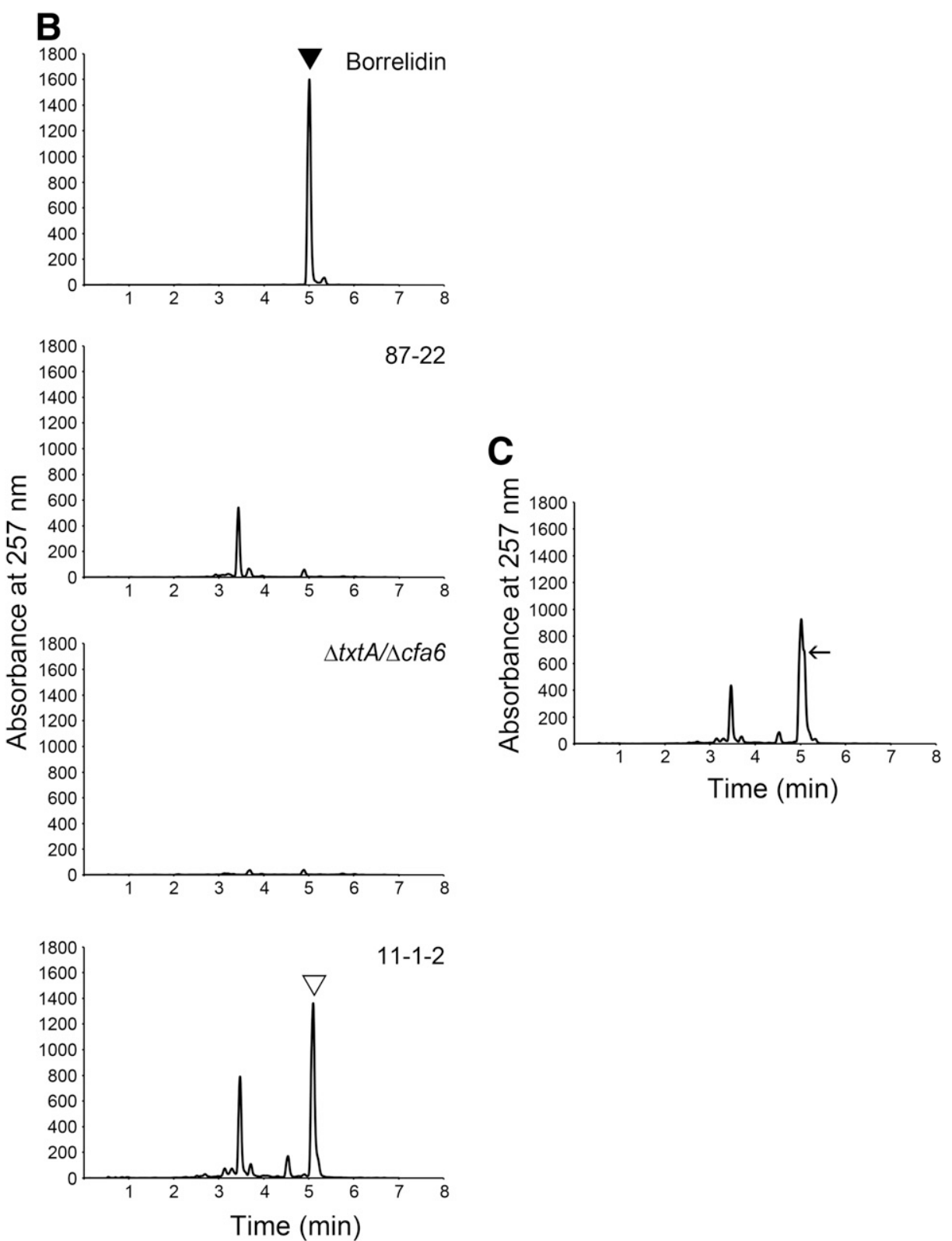

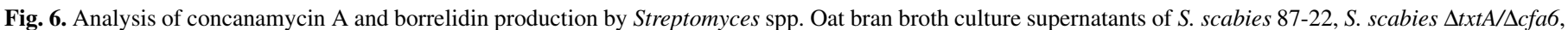

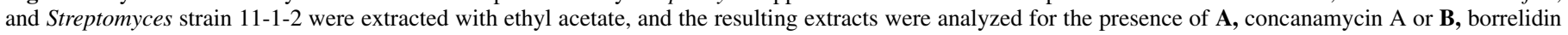

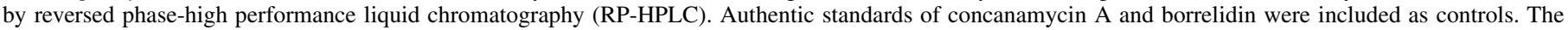

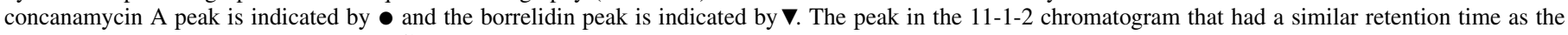

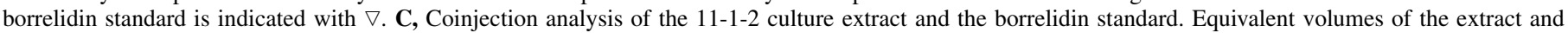

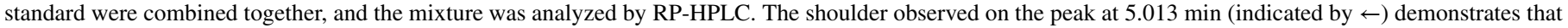
the borrelidin standard did not coelute with the $\nabla$ peak in the 11-1-2 culture extract. 
seedlings (Cao et al. 2012). We tested for the presence of borrelidin in the 11-1-2 organic culture extracts using RP-HPLC (Fig. 6B), and although an absorbance peak with a similar retention time (5.097 $\mathrm{min})$ as the borrelidin standard (5.013 $\mathrm{min}$ ) was observed in the 11-1-2 chromatogram, the two peaks did not co-elute when the standard was co-injected with the culture extract (Fig. 6C). Furthermore, LC-MS analysis of the 11-1-2 organic culture extract did not detect the presence of a compound with the same molecular mass as borrelidin (data not shown). Taken together, these results suggest that strain 11-1-2 does not produce borrelidin under the culture conditions used.

This is the first study describing the isolation and characterization of plant-pathogenic Streptomyces spp. from Newfoundland. Our results show that scab lesions on potato tubers harvested on the island are associated with a variety of pathogenic Streptomyces spp., some of which appear to be novel. The majority of pathogenic isolates were determined to be most similar to S. europaeiscabiei, which is commonly associated with CS disease in other Canadian provinces. Whether $S$. scabies and $S$. acidiscabies, which have also been reported in eastern Canada (Faucher et al. 1992; St-Onge et al. 2008; Wanner 2009), are present in Newfoundland as well remains to be determined. A particularly interesting finding from this study was the isolation of a non-thaxtomin-producing Streptomyces sp. that displays a severe pathogenic phenotype against different plant hosts and which produces a novel, secreted phytotoxic substance that is soluble in organic solvent. Further characterization of the strain and the phytotoxic substance it produces is currently underway in order to better understand how the organism is able to cause disease. Until recently, it was thought that all CS-causing pathogens produce thaxtomin $\mathrm{A}$ and that this phytotoxin is the primary virulence factor contributing to disease development; however, our findings are among a growing body of evidence suggesting that other phytotoxins can also play a role in CS disease (Bignell et al. 2014). This has important implications for the development of long-term control strategies that will effectively manage the disease in potato growing regions around the world.

\section{ACKNOWLEDGMENTS}

This work was supported by a Canada-Newfoundland and Labrador Agriculture Research Initiative grant (ARI-1314-005) to D. R. D. Bignell and by a Natural Sciences and Engineering Research Council of Canada Discovery Grant (386696-2010) to D. R. D. Bignell. We thank R.-A. Blanchard (NL Department of Natural Resources) and A. Lang (Department of Biology, Memorial University of Newfoundland) for providing the potato tubers used in this study, K. Arakawa (Hiroshima University) for kindly providing the pure borrelidin standard, and L. Windsor (Memorial University Centre for Chemical Analysis, Research and Training) for assistance with the LC-MS analysis. We also thank the anonymous reviewers for their helpful comments on the manuscript.

\section{LITERATURE CITED}

Bignell, D. R. D., Fyans, J. K., and Cheng, Z. 2014. Phytotoxins produced by plant pathogenic Streptomyces species. J. Appl. Microbiol. 116:223-235.

Bignell, D. R. D., Seipke, R. F., Huguet-Tapia, J. C., Chambers, A. H., Parry, R., and Loria, R. 2010. Streptomyces scabies 87-22 contains a coronafacic acid-like biosynthetic cluster that contributes to plant-microbe interactions. Mol. Plant-Microbe Interact. 23:161-175.

Bouarab, K., Melton, R., Peart, J., Baulcombe, D., and Osbourn, A. 2002. A saponin-detoxifying enzyme mediates suppression of plant defenses. Nature 418:889-892.

Bouchek-Mechiche, K., Gardan, L., Normand, P., and Jouan, B. 2000. DNA relatedness among strains of Streptomyces pathogenic to potato in France: Description of three new species, $S$. europaeiscabiei sp. nov. and S. stelliscabiei sp. nov. associated with common scab, and S. reticuliscabiei sp. nov. associated with netted scab. Int. J. Syst. Evol. Microbiol. 50:91-99.

Bukhalid, R. A., Chung, S. Y., and Loria, R. 1998. nec1, a gene conferring a necrogenic phenotype, is conserved in plant-pathogenic Streptomyces spp. and linked to a transposase pseudogene. Mol. Plant-Microbe Interact. 11: 960-967.
Cao, Z., Khodakaramian, G., Arakawa, K., and Kinashi, H. 2012. Isolation of borrelidin as a phytotoxic compound from a potato pathogenic Streptomyces strain. Biosci. Biotechnol. Biochem. 76:353-357.

Clark, C. A., Chen, C., Ward-Rainey, N., and Pettis, G. S. 1998. Diversity within Streptomyces ipomoeae based on inhibitory interactions, rep-PCR, and plasmid profiles. Phytopathology 88:1179-1186.

Dees, M. W., Sletten, A., and Hermansen, A. 2013. Isolation and characterization of Streptomyces species from potato scab lesions in Norway. Plant Pathol. 62:217-225.

Dees, M. W., Somervuo, P., Lysoe, E., Aittamaa, M., and Valkonen, J. P. 2012. Species' identification and microarray-based comparative genome analysis of Streptomyces species isolated from potato scab lesions in Norway. Mol. Plant Pathol. 13:174-186.

Dees, M. W., and Wanner, L. A. 2012. In search of better management of potato common scab. Potato Res. 55:249-268.

Faucher, E., Savard, T., and Bealieu, C. 1992. Characterization of actinomycetes isolated from common scab lesions on potato tubers. Can. J. Plant Pathol. 14:197-202.

Flores-González, R., Velasco, I., and Montes, F. 2008. Detection and characterization of Streptomyces causing potato common scab in Western Europe. Plant Pathol. 57:162-169.

Fyans, J. K., Altowairish, M. S., Li, Y., and Bignell, D. R. D. 2015. Characterization of the coronatine-like phytotoxins produced by the common scab pathogen Streptomyces scabies. Mol. Plant-Microbe Interact. 28:443-454.

Goyer, C., Vachon, J., and Beaulieu, C. 1998. Pathogenicity of Streptomyces scabies mutants altered in thaxtomin A production. Phytopathology 88: 442-445.

Guo, Y., Zheng, W., Rong, X., and Huang, Y. 2008. A multilocus phylogeny of the Streptomyces griseus 16S rRNA gene clade: Use of multilocus sequence analysis for streptomycete systematics. Int. J. Syst. Evol. Microbiol. 58: 149-159.

Hao, J. J., Meng, Q. X., Yin, J. F., and Kirk, W. W. 2009. Characterization of a new Streptomyces strain, DS3024, that causes potato common scab. Plant Dis. 93:1329-1334.

Healy, F. G., Wach, M., Krasnoff, S. B., Gibson, D. M., and Loria, R. 2000. The $t x t A B$ genes of the plant pathogen Streptomyces acidiscabies encode a peptide synthetase required for phytotoxin thaxtomin A production and pathogenicity. Mol. Microbiol. 38:794-804.

Hill, J., and Lazarovits, G. 2005. A mail survey of growers to estimate potato common scab prevalence and economic loss in Canada. Can. J. Plant Pathol. 27:46-52.

Hiltunen, L. H., Weckman, A., Ylhainen, A., Rita, H., Richter, E., and Valkonen, J. P. T. 2005. Responses of potato cultivars to the common scab pathogens, Streptomyces scabies and S. turgidiscabies. Ann. Appl. Biol. 146:395-403.

Ito, S., Eto, T., Tanaka, S., Yamauchi, N., Takahara, H., and Ikeda, T. 2004. Tomatidine and lycotetraose, hydrolysis products of alpha-tomatine by Fusarium oxysporum tomatinase, suppress induced defense responses in tomato cells. FEBS Lett. 571:31-34.

Johnson, E. G., Joshi, M. V., Gibson, D. M., and Loria, R. 2007. Cellooligosaccharides released from host plants induce pathogenicity in scabcausing Streptomyces species. Physiol. Mol. Plant Pathol. 71:18-25.

Joshi, M., Rong, X., Moll, S., Kers, J., Franco, C., and Loria, R. 2007a. Streptomyces turgidiscabies secretes a novel virulence protein, $\mathrm{Nec} 1$, which facilitates infection. Mol. Plant-Microbe Interact. 20:599-608.

Joshi, M. V., Bignell, D. R., Johnson, E. G., Sparks, J. P., Gibson, D. M., and Loria, R. 2007b. The AraC/XylS regulator TxtR modulates thaxtomin biosynthesis and virulence in Streptomyces scabies. Mol. Microbiol. 66: 633-642.

Kers, J. A., Cameron, K. D., Joshi, M. V., Bukhalid, R. A., Morello, J. E., Wach, M. J., Gibson, D. M., and Loria, R. 2005. A large, mobile pathogenicity island confers plant pathogenicity on Streptomyces species. Mol. Microbiol. 55:1025-1033.

Kieser, T., Bibb, M. J., Buttner, M. J., Chater, K. F., and Hopwood, D. A. 2000. Practical Streptomyces. Genetics. The John Innes Foundation, Norwich, U.K.

Kim, Y., Cho, J. M., Park, D., Lee, H., Kim, J., Seo, S., Shin, K., Hur, J., and Lim, C. 1999. Production of thaxtomin A by Korean isolates of Streptomyces turgidiscabies and their involvement in pathogenicity. Plant Pathol. J. 15:168-171.

Kinashi, H., Someno, K., and Sakaguchi, K. 1984. Isolation and characterization of concanamycins A, B and C. J. Antibiot. 37:1333-1343.

King, R. R., and Calhoun, L. A. 2009. The thaxtomin phytotoxins: Sources, synthesis, biosynthesis, biotransformation and biological activity. Phytochemistry 70:833-841.

Kreuze, J. F., Suomalainen, S., Paulin, L., and Valkonen, J. P. 1999. Phylogenetic analysis of 16SrRNA genes and PCR analysis of the necl gene from Streptomyces spp. causing common scab, pitted scab, and netted scab in Finland. Phytopathology 89:462-469. 
Lambert, D. H., and Loria, R. 1989a. Streptomyces acidiscabies sp. nov. Int. J. Syst. Bacteriol. 39:393-396.

Lambert, D. H., and Loria, R. 1989b. Streptomyces scabies sp. nov., nom. rev. Int. J. Syst. Bacteriol. 39:387-392.

Lehtonen, M. J., Rantala, H., Kreuze, J. F., Bang, H., Kuisma, L., Koski, P., Virtanen, E., Vihlman, K., and Valkonen, J. P. T. 2004. Occurrence and survival of potato scab pathogens (Streptomyces species) on tuber lesions: Quick diagnosis based on a PCR-based assay. Plant Pathol. 53: 280-287.

Loria, R., Bignell, D. R., Moll, S., Huguet-Tapia, J. C., Joshi, M. V., Johnson, E. G., Seipke, R. F., and Gibson, D. M. 2008. Thaxtomin biosynthesis: The path to plant pathogenicity in the genus Streptomyces. Antonie van Leeuwenhoek 94:3-10.

Loria, R., Bukhalid, R. A., Creath, R. A., Leiner, R. H., Olivier, M., and Steffens, J. C. 1995. Differential production of thaxtomins by pathogenic Streptomyces species in vitro. Phytopathology 85:537-541.

Loria, R., Bukhalid, R. A., Fry, B. A., and King, R. R. 1997. Plant pathogenicity in the genus Streptomyces. Plant Dis. 81:836-846.

Loria, R., Kers, J., and Joshi, M. 2006. Evolution of plant pathogenicity in Streptomyces. Annu. Rev. Phytopathol. 44:469-487.

Miyajima, K., Tanaka, F., Takeuchi, T., and Kuninaga, S. 1998. Streptomyces turgidiscabies sp. nov. Int. J. Syst. Bacteriol. 48:495-502.

Natsume, M., Ryu, R., and Abe, H. 1996. Production of phytotoxins, concanamycins A and B by Streptomyces spp. causing potato scab. Ann. Phytopathol. Soc. Jpn. 62:411-413.

Natsume, M., Yamada, A., Tashiro, N., and Abe, H. 1998. Differential production of the phytotoxins thaxtomin A and concanamycins A and B by potato common scab-causing Streptomyces spp. Ann. Phytopathol. Soc. Jpn. 64:202-204.

Olano, C., Moss, S. J., Braña, A. F., Sheridan, R. M., Math, V., Weston, A. J., Méndez, C., Leadlay, P. F., Wilkinson, B., and Salas, J. A. 2004. Biosynthesis of the angiogenesis inhibitor borrelidin by Streptomyces parvulus Tü4055: Insights into nitrile formation. Mol. Microbiol. 52:1745-1756.

Park, D. H., Kim, J. S., Kwon, S. W., Wilson, C., Yu, Y. M., Hur, J. H., and Lim, C. K. 2003. Streptomyces luridiscabiei sp. nov., Streptomyces puniciscabiei sp. nov. and Streptomyces niveiscabiei sp. nov., which cause potato common scab disease in Korea. Int. J. Syst. Evol. Microbiol. 53: 2049-2054.

Seipke, R. F., and Loria, R. 2008. Streptomyces scabies 87-22 possesses a functional tomatinase. J. Bacteriol. 190:7684-7692.

Song, J., Lee, S. C., Kang, J. W., Baek, H. J., and Suh, J. W. 2004. Phylogenetic analysis of Streptomyces spp. isolated from potato scab lesions in Korea on the basis of 16S rRNA gene and 16S-23S rDNA internally transcribed spacer sequences. Int. J. Syst. Evol. Microbiol. 54:203-209.

St-Onge, R., Goyer, C., Coffin, R., and Filion, M. 2008. Genetic diversity of Streptomyces spp. causing common scab of potato in eastern Canada. Syst. Appl. Microbiol. 31:474-484.

Tamura, K., Peterson, D., Peterson, N., Stecher, G., Nei, M., and Kumar, S. 2011. MEGA5: Molecular evolutionary genetics analysis using maximum likelihood, evolutionary distance, and maximum parsimony methods. Mol. Biol. Evol. 28:2731-2739.

Thwaites, R., Wale, S. J., Nelson, D., Munday, D., and Elphinstone, J. G. 2010. Streptomyces turgidiscabies and $S$. acidiscabies: Two new causal agents of common scab of potato (Solanum tuberosum) in the UK. Plant Pathol. 59: 804.

Tóth, L., Maeda, M., Tanaka, F., and Kobayashi, K. 2001. Isolation and identification of pathogenic strains of Streptomyces acidiscabies from netted scab lesions of potato tubers in Hokkaido (Japan). Acta Microbiol. Immunol. Hung. 48:575-585.

VanEtten, H. D., Mansfield, J. W., Bailey, J. A., and Farmer, E. E. 1994. Two classes of plant antibiotics: phytoalexins versus "phytoanticipins". Plant Cell 6:1191-1192.

Wanner, L. A. 2004. Field isolates of Streptomyces differ in pathogenicity and virulence on radish. Plant Dis. 88:785-796.

Wanner, L. A. 2007. A new strain of Streptomyces causing common scab in potato. Plant Dis. 91:352-359.

Wanner, L. A. 2009. A patchwork of Streptomyces species isolated from potato common scab lesions in North America. Am. J. Potato Res. 86:247-264.

Zhao, W. Q., Liu, D. Q., and Yu, X. M. 2008. First report of potato scab caused by Streptomyces turgidiscabies in China. Plant Dis. 92:1587.

Zhao, W. Q., Yu, X. M., and Liu, D. Q. 2010. First report of Streptomyces acidiscabies causing potato scab in China. Plant Pathol. 59:405. 